\title{
Recurrent Tracheoinnominate Artery Fistula due to Stent Graft Fracture
}

\author{
Hyo Yeong Ahn ${ }^{1}$ Bong Soo Son ${ }^{2}$ Do Hyung Kim² \\ ${ }^{1}$ Department of Thoracic and Cardiovascular Surgery, Amidong Seo-gu \\ Pusan National University Hospital, Busan, Republic of Korea \\ 2 Department of Thoracic and Cardiovascular Surgery, Pusan National \\ University Yangsan Hospital, Yangsan, Republic of Korea \\ Eur J Pediatr Surg Rep 2015;3:12-14.
}

Address for correspondence Do Hyung Kim, MD, PhD, Department of Thoracic and Cardiovascular Surgery, Pusan National University Yangsan Hospital, Yangsan, Republic of Korea (e-mail: yumccs@nate.com).
Abstract
Keywords
- tracheoinnominate artery fistula (TIF)
- endovascular stent graft repair
- stent graft fracture

Tracheoinnominate artery fistula (TIF) is one of the most dangerous complications after tracheostomy, and fetal even after surgical repair. Recently, endovascular stent has been introduced as an option for the treatment of TIF. Unfortunately, endovascular stent repair could not replace the surgery due to rare clinical reports about the long-term follow-up and complications of stent graft. More collection of clinical data are necessary for the evaluation of long-term results. We report a rare case of recurrent TIF due to stent graft fracture after endovascular stent graft insertion in the treatment of TIF, and reviewed the world literature.

\section{Introduction}

Although the incidence of tracheoinnominate artery fistula (TIF) has decreased, it is still one of the most dangerous complications after tracheostomy. Almost $75 \%$ of patients die from a TIF, even after surgical repair has been performed. Therefore, it is doubtful whether surgical repair is an effective method for treating TIF; however, it is still considered to be the optimal treatment option for TIF.

Recently, the endovascular intervention has become the first-line treatment modality for vascular diseases and has replaced the classical surgical approach in many areas. In some cases of TIF, endovascular stent graft repair (ESGR) was successfully used as the first-line treatment. ${ }^{1}$ As the longterm outcomes and complications of TIF endovascular intervention have been rarely reported, it is still wondered that the endovascular intervention can be treated as a better modality than surgery. Here, we present a rare case of recurrent TIF due to stent graft fracture after ESGR.

\section{Case Report}

A 14-year-old male patient who had been followed-up for cerebral palsy and epilepsy from birth was admitted to the emergency room with fever, increase in the cough and sputum, and fresh bloody secretion through the tracheostomy. The patient had history of TIF developed in 10 days after tracheostomy and was treated with endovascular stent graft (JO stent graft, JOMED GmbH, Rangendmgen, Germany; $12 \mathrm{~mm} / 58 \mathrm{~mm}$ ) insertion of the innominate artery.

On the initial chest X-ray, migrated stent fragments could be seen and stent fragmentation was presumed to be caused by stent graft fracture ( - Fig. 1 ). Hence, chest computed tomography was performed, and it revealed separate stent fragments in the innominate artery that was filled by thrombus. As the bloody secretion through the tracheostomy increased, an emergent operation was planned.

Under general anesthesia, the sternotomy was performed. Initially, the proximal and distal portions of the innominate artery were dissected and ligated with sutures, and then the inflammatory and necrotic tissues around the TIF were resected. The migrated stent fragments were identified and removed through the TIF ( - Fig. 2). The inflammatory lesion in the tracheal defect was excised and the end-to-end anastomosis was performed. The patient was given ventilatory support for 4 days, and he was transferred to the general ward on the 7th postoperative day (POD) and discharged on the 33th POD. received

November 4, 2013

accepted

December 31, 2013

published online

September 3, 2014

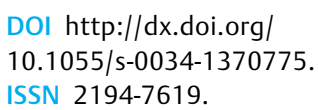

DOI http://dx.doi.org/

10.1055/s-0034-1370775. ISSN 2194-7619.

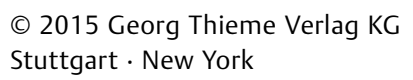

License terms

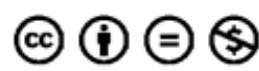




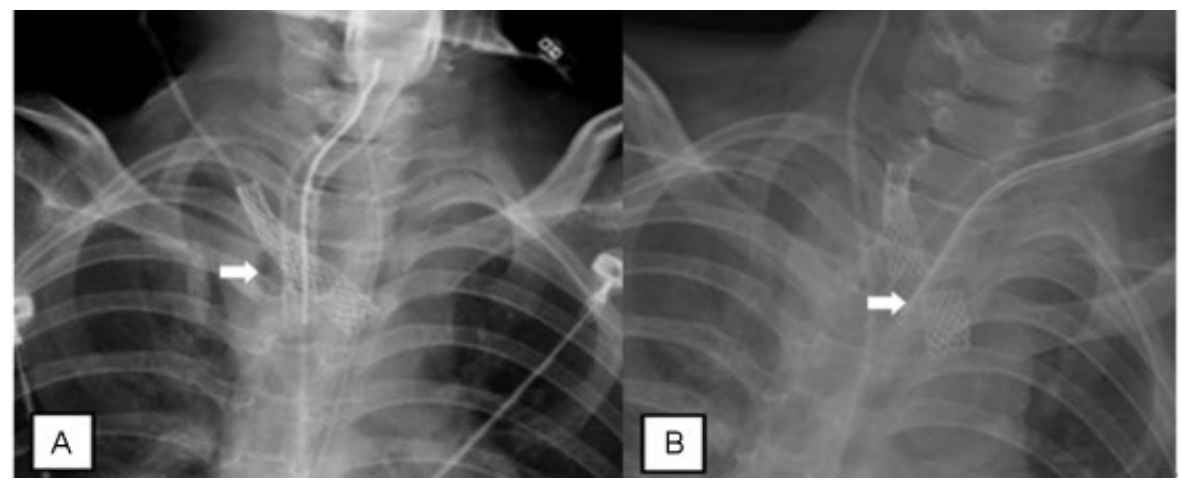

Fig. 1 (A) Initial chest X-ray after endovascular stent graft repair (arrow) of the innominate artery. (B) Chest X-ray shows the migrated stent fragments (arrow) that developed due to stent graft fracture

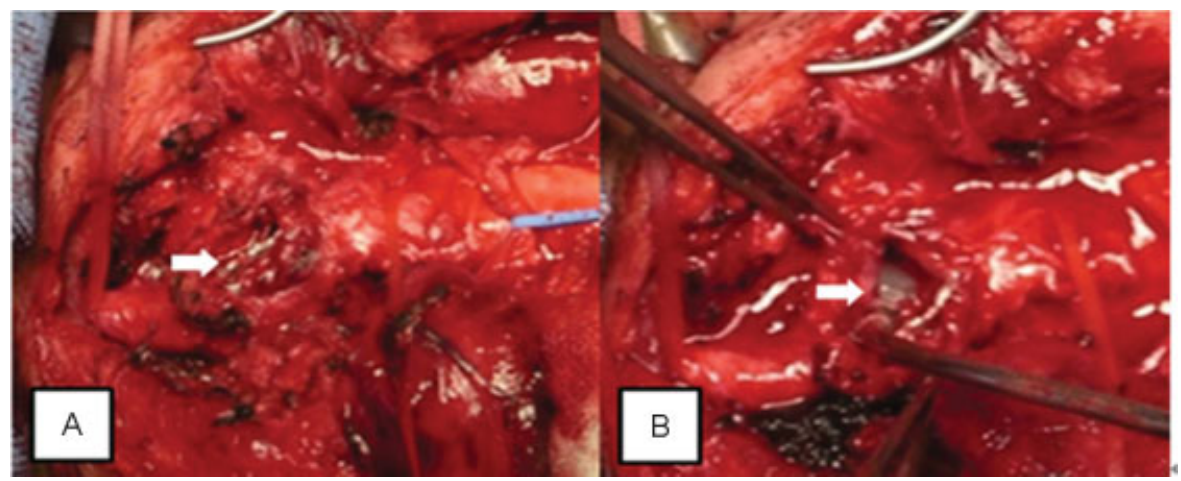

Fig. 2 (A) The endovascular stent fragments (arrow) could be seen through a tear in the innominate artery. (B) After placing the innominate artery laterally, the endotracheal tube (arrow) could be seen through the fistula in trachea.

\section{Discussion}

Since Deguch et al successfully performed ESGR in a patient with TIF, 14 TIF cases, including our case, that were treated by endovascular intervention have been reported until 2012 in the world literature. ${ }^{1-7}$ Most endovascular interventions were performed as the first-line treatment, except one case, in which endovascular intervention was performed as a bridge to surgical treatment. ${ }^{8}$ Considering the fact that bleeding could be controlled in most of cases, ESGR seems to be an optimal treatment option for a TIF. ${ }^{2-5}$ However, based on different individual situations, ESGR cannot be considered to be superior to surgical repair and should be prudently considered depending on the case.

To date, postinterventional complications have occurred in six cases including our case. Three cases immediately developed endoleak after stent graft insertion, and three cases were recurrent TIF's which were stent-related complications. Of the three cases of endoleak, one case was recovered after operation and the other case was recovered after an additional ESGR. However, the third patient died from hemorrhage even though an additional ESGR was performed. Of the three recurrent TIF's, two cases were developed due to aggravation of fistula by continuous pressure of the inserted stent, and one case due to stent graft fracture as mentioned in our case. First two patients died from rebleeding even though the operation was performed. ${ }^{4-8}$

Although there were several reports of successful management in TIF patient who underwent ESGR, ${ }^{1,2,7}$ it is still difficult to decide whether ESGR for TIF is a permanent treatment of option due to critical postinterventional complications. As the long-term outcome of ESGR in TIF has been unclear, ESGR should be prudently performed in a child or an adolescent who has a long life expectancy. In patient with life-threatening bleeding from TIF, ESGR could be a good treatment of option as a bridge to surgical treatment. Besides, the planed surgical correction is the most ideal approach for dealing with postinterventional complications.

\section{Conflict of Interest}

None.

\section{References}

1 Deguchi J, Furuya T, Tanaka N, et al. Successful management of tracheo-innominate artery fistula with endovascular stent graft repair. J Vasc Surg 2001;33(6):1280-1282 
14 Recurrent TIF due to Stent Graft Fracture Ahn et al.

2 Shepard PM, Phillips JM, Tefera G, Hartig GK. Tracheoinnominate fistula: successful management with endovascular stenting. Ear Nose Throat J 2011;90(7):310-312

3 Wang PK, Yen PS, Shyr MH, Chen TY, Chen A, Liu HT. Endovascular repair of tracheo-innominate artery fistula. Acta Anaesthesiol Taiwan 2009;47(1):36-39

4 Sorial E, Valentino J, Given CA, Endean ED, Minion DJ. The emergency use of endografts in the carotid circulation to control hemorrhage in potentially contaminated fields. J Vasc Surg 2007; 46(4):792-798

5 Sessa C, Costache V, Porcu P, et al. Tracheoinnominate artery fistula: combined endovascular and surgical management by emergency stent-graft placement followed by cryopreserved arterial allograft repair. Ann Vasc Surg 2006;20(6):731-735

6 Wall LP, Gasparis A, Criado E. Endovascular therapy for tracheoinnominate artery fistula: a temporizing measure. Ann Vasc Surg 2005;19(1):99-102

7 Vianello A, Ragazzi R, Mirri L, Arcaro G, Cutrone C, Fittà C. Tracheoinnominate fistula in a Duchenne muscular dystrophy patient: successful management with an endovascular stent. Neuromuscul Disord 2005;15(8):569-571

8 Marone EM, Esposito G, Kahlberg A, et al. Surgical treatment of tracheoinnominate fistula after stent-graft implantation. J Thorac Cardiovasc Surg 2007;133(6):1641-1643 